\title{
Subacute painless bilateral visual loss
}

\author{
Joseph Habboushe $\cdot$ Kamal Medlej • \\ Kaushal Shah
}

Received: 10 February 2010/Accepted: 24 February 2010/Published online: 21 April 2010

(C) SIMI 2010

\section{Case synopsis}

A 67-year-old man with a history of CHF (ejection fraction of $10 \%$ on transthoracic echocardiogram) presented to the ED with progressive bilateral painless visual loss over the prior $6 \mathrm{~h}$. The patient had been discharged from another hospital approximately $8 \mathrm{~h}$ prior to arrival, after a weeklong inpatient stay for exacerbation of CHF. While being driven home after hospital discharge, he began to notice a bilateral blurring of his vision to the point of only being able to differentiate light from dark. This prompted him to go directly to his ophthalmologist where examination revealed a normal pupillary response, normal funduscopy, and no clear etiology for the visual loss. The patient was told to come directly to the ED to rule out a stroke. On arrival at the ED, the patient denied having eye pain, headache, focal motor weakness or numbness, slurring of his speech, or other new symptoms. The patient was found to be in rapid atrial fibrillation at a rate of 165 beats $/ \mathrm{min}$. The rest of the vital signs were: blood pressure 123/ $94 \mathrm{mmHg}$, respiratory rate 16 breaths/min, oral temperature $37.8^{\circ} \mathrm{C}\left(100^{\circ} \mathrm{F}\right.$,) oxygen saturation $99 \%$ (on nasal cannula $3 \mathrm{~L} / \mathrm{min}$ ). He denied chest pain, shortness of breath, palpitations, or dizziness. He was given two doses of diltiazem $10 \mathrm{mg}$ intravenously, but the heart rate remained elevated. It was finally controlled with an esmolol drip. A computed tomography of the brain was obtained (Fig. 1).

J. Habboushe $(\bowtie) \cdot$ K. Medlej $\cdot$ K. Shah

Department of Emergency Medicine, St. Luke's-Roosevelt

Hospital of Columbia University, 1000 Tenth Avenue,

New York, NY 10019, USA

e-mail: joehabb@yahoo.com

\section{Background}

Cortical blindness is the complete loss of vision due to bilateral occipital lobe lesions. Cortical blindness presents with visual loss and limited eyelid closure response to bright light, but a normal pupillary response and normal funduscopic examination. The majority of those presenting acutely are due either to sudden and severe blood loss, hypertensive crisis, or toxemia of pregnancy [1]. Uncommonly trauma and tumors can be the etiology, as well as PCA compression along the tentorium due to increased intracerebral pressures in patients with predisposed anatomy, although this often causes a concomitant motor impairment [2]. The Anton-Babinski Syndrome describes the poorly understood state of denial of one's blindness sometimes seen in patients with cortical blindness. The Riddoch Phenomenon is the ability to sense movement but remain blind to the static, often seen in patients recovering from cortical blindness. [1] Most unilateral cortical lesions are due to emboli from the heart or a vertebro-basilar syndrome [3].

\section{Diagnosis and discussion}

Our patient was presumed to have bilateral embolic infarcts due to the atrial fibrillation, which we learned was of newonset since he was discharged in sinus rhythm earlier that day. However, the patient did report a history of past atrial fibrillation, along with congestive heart failure. He had been on warfarin that had been recently discontinued due to a history of gastrointestinal bleeding. The presumed embolic strokes likely became hemorrhagic, leading to the subacute bilateral blindness he presented with over several hours. Our patient did not present with the Anton-Babinski 


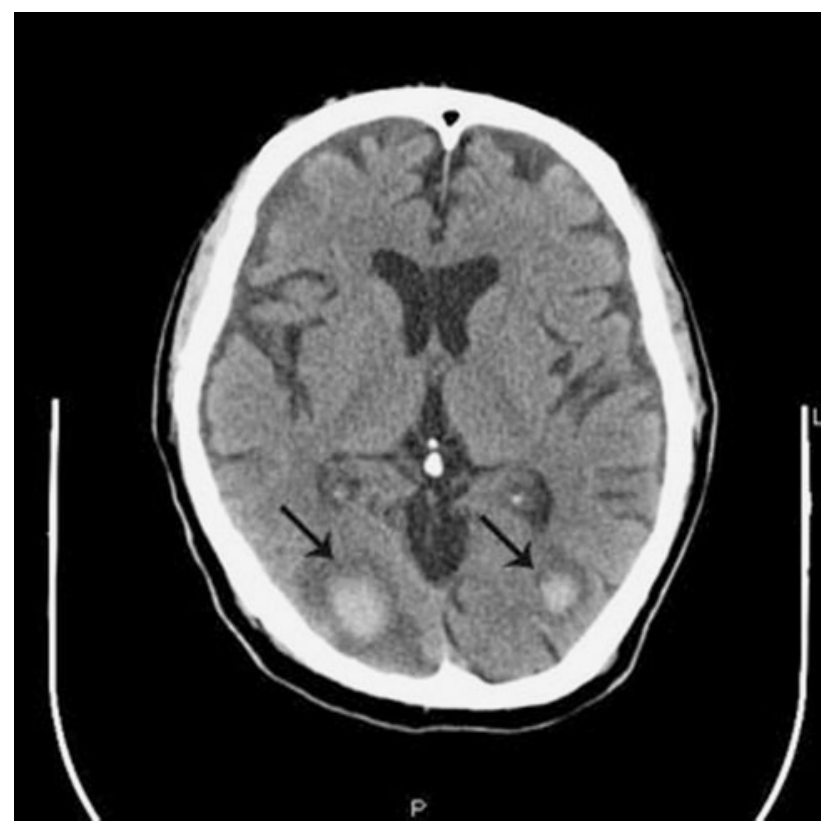

Fig. 1 Non-contrast computed tomography of the brain showing bilateral occipital lobe intraparenchymal hemorrhages with mass effect

Syndrome nor was Riddoch's phenomenon clearly elicited, although the patient could recognize light versus dark.

After the diagnosis of bilateral cortical hemorrhage was made by computed tomography, the Stroke service was consulted, and in accord with their recommendation, platelets were transfused, and the esmolol drip was continued for heart rate and blood pressure control. A second computed tomography, this time with intravenous contrast, was also performed to rule out thrombosis, and the patient was admitted to the neurological ICU. At the time of admission, the patient was not experiencing any improvement in symptoms. The patient remained in the neurologic ICU for 3 days during which time his vision improved bilaterally to the point of distinguishing shapes and colors. A left homonymous hemianopsia persisted. The patient left the unit against medical advice on the third day of admission.

Conflict of interest None.

\section{References}

1. Lee D, Higginbothan E (1999) Clinical guide to comprehensive ophthalmology. Thieme Medical Publisher, New York, pp 521522

2. Brandt $\mathrm{T}$ et al (2000) Posterior cerebral artery territory infarcts: clinical features, infarct topography, causes and outcomes. Cerebrovasc Dis 10:170-182

3. Nehmud L (1998) The end in sight: a look at the occipital lobe. Clin Eye Vis Care 10:125-133 